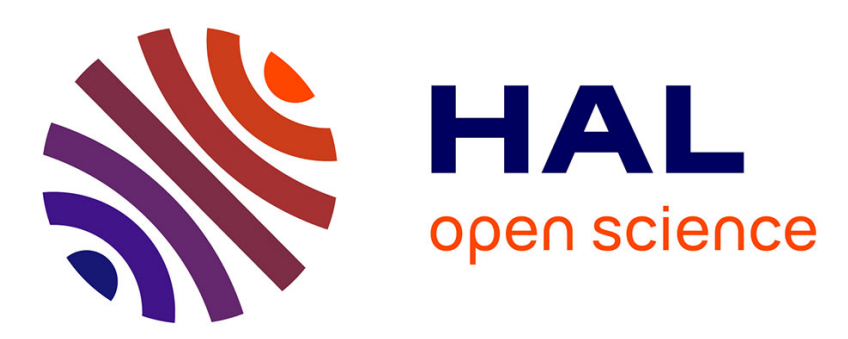

\title{
Modèles de prévision et de simulation de l'irradiation solaire au pas de temps de l'heure
}

\author{
R. Astier, Ch. Duhamel, C. Bénard
}

\section{To cite this version:}

R. Astier, Ch. Duhamel, C. Bénard. Modèles de prévision et de simulation de l'irradiation solaire au pas de temps de l'heure. Revue de Physique Appliquée, 1983, 18 (1), pp.29-38. 10.1051/rphysap:0198300180102900 . jpa-00245065

\section{HAL Id: jpa-00245065 https://hal.science/jpa-00245065}

Submitted on 1 Jan 1983

HAL is a multi-disciplinary open access archive for the deposit and dissemination of scientific research documents, whether they are published or not. The documents may come from teaching and research institutions in France or abroad, or from public or private research centers.
L'archive ouverte pluridisciplinaire HAL, est destinée au dépôt et à la diffusion de documents scientifiques de niveau recherche, publiés ou non, émanant des établissements d'enseignement et de recherche français ou étrangers, des laboratoires publics ou privés. 


\title{
Modèles de prévision et de simulation de l'irradiation solaire au pas de temps de l'heure
}

\author{
R. Astier, Ch. Duhamel \\ Département de Statistiques, Bât. de Mathématiques (425), Université Paris XI, 91405 Orsay, France \\ et C. Bénard \\ CNRS, Ecole Supérieure d'Electricité, Laboratoire des Signaux et Systèmes, \\ Plateau du Moulon, 91190 Gif sur Yvette, France
}

(Reçu le 2 juillet 1982, révisé le 15 octobre 1982, accepté le 20 octobre 1982)

\begin{abstract}
Résumé. - Une étude statistique des données météorologiques de Trappes conduit à un modèle de prévision de l'irradiation solaire globale de ce site au pas de temps de l'heure et montre la stabilité du modèle au cours de l'année. On étudie ensuite l'évolution de cette irradiation conditionnellement à celle de la nébulosité mesurée toutes les trois heures. On aboutit ainsi à une simulation simple de l'irradiation à partir d'une pré-simulation des nébulosités par un modèle semi-markovien.

Abstract. - On the basis of the meteorological data from Trappes (France) we build a prediction model of the total solar irradiation for a one-hour-step, and we show that this model is stable all the year round.

The evolution of the hourly irradiation conditionally to that of the nebulosity measured every three hours is also studied. This leads to a simple simulation model of the total solar irradiation built on a pre-simulation of the nebulosity by a semi-markovian model.
\end{abstract}

1. Introduction. - La conception, le dimensionnement et la gestion optimale de systèmes énergétiques solaires passent impérativement par la connaissance des conditions météorologiques auxquelles sont soumis ces systèmes. Celle-ci doit s'appuyer sur des méthodes statistiques relativement fines. En effet, la conception et les dimensions d'un système ne dépendent des valeurs moyennes, saisonnières quotidiennes ou horaires, des variables météorologiques qu'en première approximation. Les fluctuations aléatoires de ces dernières, au cours du temps, contraignent les systèmes à fonctionner en régime instationnaire et la mémoire de ces fluctuations influence grandement les dimensions optimales du système. De plus, l'optimisation de la gestion d'un système énergétique solaire suppose que l'on dispose de modèles de prévision des variables météorologiques qui associés à un modèle adéquat du système étudié et aux critères d'optimisation que l'on s'impose permettent l'établissement de commandes.

L'ensemble de ces raisons nous amène à construire des modèles stochastiques des variables météorologiques pour la simulation (conception, dimensionnement) ou la prévision (gestion) aussi aisés que possible à manipuler et condensant donc l'information à l'aide de quelques paramètres et lois de probabilité simples.
C'est dans cet optique que nous avons déjà développé des modèles stochastiques temporels de l'irradiation solaire d'un site au pas de temps de la journée [1] et de l'heure [2]. Les modèles utilisés sont des modèles ARMA [3] de la variable réduite irradiation solaire, c'est-à-dire d'une variable déduite de la variable originelle par élimination des variations saisonnières de la moyenne et de l'écart quadratique. Ces modèles sont une approximation d'une équation satisfaite par tout processus stationnaire du second ordre mesuré à des intervalles de temps régulièrement espacés. Ils expriment la variable considérée $z_{t}$ comme combinaison linéaire finie d'une part de ses valeurs à des instants antérieurs, d'autre part des valeurs d'un bruit blanc $\left(e_{t}\right)$ à des temps inférieurs ou égaux à $t$. Les modèles ARMA, par opposition à des chaînes de Markov par exemple, présentent l'intérêt de faire prendre à la variable ses valeurs sur un espace continu, respectant ainsi ce caractère physique important des variables qui nous intéressent, telles que l'irradiation, ou la température.

Ces modèles ont mis en évidence la faible mémoire d'un modèle monovariable de l'irradiation au pas de temps quotidien : les coefficients de corrélation de l'irradiation, d'un jour à l'autre sont de l'ordre de 0,3 .

L'analyse des corrélations linéaires entre plusieurs 
variables réduites météorologiques d'un même site, au pas de temps quotidien, a mis en évidence, sur le site de Trappes, la forte corrélation du groupe de variables Irradiation-Nébulosité-Humidité relative (coefficients d'intercorrélation deux à deux, le même jour, de l'ordre de 0,8 ; à un jour d'intervalle, de l'ordre de 0,25 ). Les autres variables du site (température, force et direction du vent, pression) sont nettement moins corrélées [4]. Ces résultats montrent que, sur une même variable et entre variables fortement corrélées, la corrélation décroît très vite sur 24 heures et que pour améliorer la prévision sur l'irradiation, il faut passer à un pas de temps plus faible. C'est en ce sens qu'un modèle de régression de l'irradiation quotidienne sur la nébulosité du matin (de 6 heures à 9 heures) a été établi [4]. C'est également en ce sens qu'un modèle de l'irradiation horaire a été établi qui rend possible la prévision de l'irradiation d'une heure à l'autre : en effet les coefficients d'autocorrélation d'une heure à l'autre sont de l'ordre de 0,80 à 0,85 [2].

Dans la présente étude, dans le but d'améliorer la modélisation de l'irradiation en un site, nous avons repris l'analyse multivariable, au pas de temps de l'heure, sur un ensemble des variables suivantes : irradiation, température, humidité relative, pression et nébulosité. Plus précisément notre étude vise à obtenir deux modèles :

- prévision de l'irradiation globale au pas de temps horaire en tenant compte d'autres variables mesurées à l'aide d'appareils enregistreurs : température, humidité relative et pression atmosphérique,

- simulation de l'évolution au cours de plusieurs journées successives de cette irradiation, en tenant compte éventuellement d'une ou plusieurs variables très explicatives (en l'occurrence la nébulosité, mesurée à l'œil nu).

Les données de base que nous avons utilisées sont fournies par le centre de la météorologie nationale de Trappes (France) pour les douze années de 1965 à 1976. On distingue deux types de données :

- les données trihoraires mesurées chaque jour à $0,3,6,9,12,15,18$ et 21 heures $t_{\mathrm{u}}$ (temps universel). Elles concernent la température, l'humidité, la pression et la nébulosité notées respectivement pour les mesures à l'heure $t: T_{t}, H_{t}, P_{t}, N_{t}$,

- les données horaires, mesurées en heures tsv (temps solaire vrai). Elles concernent l'irradiation globale $Z_{t}$ reçue sur une surface-unité entre les heures $t-1$ et $t$, pour $t$ variant de 5 à 20 heures tsv.

2. Linéarité des dépendances - On compare le coefficient de corrélation linéaire $\rho_{X Y}$ au rapport $R_{X Y}$. Par définition :

$$
\begin{aligned}
\rho_{X Y}^{2} & =\frac{\operatorname{Min}_{a, b} \mathrm{E}[Y-(a+b X)]^{2}}{\mathrm{E}[Y-\mathrm{E}(Y)]^{2}} ; \\
R_{X Y}^{2} & =\frac{\mathrm{E}[Y-\mathrm{E}(Y / X)]^{2}}{\mathrm{E}[Y-\mathrm{E}(Y)]^{2}} .
\end{aligned}
$$

L'espérance conditionnelle $\mathrm{E}(Y / X=x)$ est estimée à partir d'une estimation non paramétrique de la densité du couple de variables aléatoires $(X, Y)$ (voir [5]).

On rappelle que $R^{2} \geqslant \rho^{2}$ et que la régression est linéaire si et seulement si $R^{2}=\rho^{2}$ (voir [6]).

On a notamment les résultats suivants (Tableau I), en prenant comme variable $Y$ l'irradiation à 14 heures, $Z_{14}$, pour la période du 10 au 30 juin (12 années). On voit que, pour ces résultats, $R^{2} \simeq \rho^{2}$.

Tableau I. - Coefficient de corrélation $\rho_{X Y}$ et rapport de corrélation $R_{X Y}$.

[Correlation coefficient $\rho_{X Y}$ and correlation ratio $R_{X Y}$.]

\begin{tabular}{|ccccc|}
\hline Variable $X$ & $Z_{13}$ & $Z_{12}$ & $T_{12}$ & $H_{12}$ \\
\hline$\left|\rho_{X Y}\right|$ & 0,81 & 0,78 & 0,46 & 0,67 \\
$R_{X Y}$ & 0,83 & 0,80 & 0,51 & 0,68 \\
\hline
\end{tabular}

On montre d'autre part que l'on peut considérer (test de Kolmogorov-Smirnov [7]) que les résidus des régressions linéaires sont gaussiens. D'autres calculs sur d'autres heures donnent sensiblement les mêmes résultats : pour les couples de variables continues $(X, Y)$ considérées, on peut admettre la linéarité des dépendances et la normalité des résidus des régressions.

3. Modèle de prévision de l'irradiation. - Pour chacune des heures $t=6,9,12$ et 15 , on considère les données : $Z_{t-1}, Z_{t}, Z_{t+1}, T_{t}, H_{t}, P_{t}, N_{t}$. On considérera également la nébulosité $N_{t+3}$ en vue d'une utilisation ultérieure. Chaque étude est réalisée à l'aide des données de trois semaines (ex. du 10 au 30 juin) répétées sur 12 années. Pour chaque étude et pour chaque heure $t$, on a donc en principe $21 \times 12=252$ données. A toute variable $V_{t}$ (sauf les nébulosités) on associe la variable centrée réduite $v_{t}=\frac{V_{t}-\bar{V}_{t}}{\sigma\left(V_{t}\right)}$, où $\bar{V}_{t}$ et $\sigma\left(V_{t}\right)$ sont les estimés usuels de la moyenne et de l'écart-type à l'heure $t$ ( 252 répétitions). Vues les faibles différences, qui évoluent au cours de l'année, entre les heures $t_{\mathrm{u}}$ et $t_{\mathrm{sv}}$, nous n'avons pas fait de correction, mais il est cependant certain que cela affecte les corrélations entre irradiation et variables trihoraires, dans la mesure où les écarts peuvent atteindre la demi-heure.

L'étude sur trois semaines est répétée au cours de l'année. On remarque qu'en considérant les variables centrées réduites pour chaque heure $t$, les dépendances de l'irradiation et des autres variables varient peu à la fois au cours de la journée et suivant la période considérée dans l'année.

3.1 RÉGRESSION PAR HEURES SÉPARÉES (H.S.). L'heure $t$ étant fixée ( $t=6,9,12$ ou 15), on considère 
le vecteur centré réduit $\left(z_{t-1}, z_{t}, z_{t+1}, h_{t}, \tau_{t}, p_{t}\right)$ où les variables réduites sont : $z=$ irradiation, $h=$ humidité, $\tau=$ température, $p=$ pression. On étudie les régressions linéaires suivantes :

$$
\begin{aligned}
& z_{t+1}=\rho_{t} z_{t}+\varepsilon_{1, t+1} \\
& z_{t+1}=a_{t} z_{t}+b_{t} x_{t}+\varepsilon_{2, t+1}
\end{aligned}
$$

où $x_{t}$ est la variable choisie, en plus de $z_{t}$ et parmi $h_{t}$,

Tableau II. - Pour chaque période figurent :

à heure $t$ fixée $: \begin{array}{l}\rho\left(z_{t}, z_{t+1}\right) \\ \sigma_{2}^{2}(\text { équation } 2), x_{t}\end{array} \quad$ à heure confondue $\left.: \begin{array}{l|l|l|}\sigma_{1}^{2} & \sigma_{2}^{2}, x \\ \sigma_{3}^{2} & \sigma_{4}^{2}\end{array}\right]$

où $\sigma_{1}^{2}, \sigma_{2}^{2}, \sigma_{3}^{2}, \sigma_{4}^{2}$ se réfèrent aux équations (1), (2), (3), (4) ci-dessous.

[For every period :

at a given time : $\begin{aligned} & \rho\left(z_{t}, z_{t+1}\right) \\
& \sigma_{2}^{2}\left(\text { Eq. 2), } x_{t}\right.\end{aligned} \quad$ for the whole set of times : \begin{tabular}{|l|l|}
$\sigma_{1}^{2}$ & $\sigma_{2}^{2}, x$ \\
$\sigma_{3}^{2}$ & $\sigma_{4}^{2}$
\end{tabular}

\begin{tabular}{|c|c|c|c|c|c|c|c|c|c|}
\hline \multirow[b]{2}{*}{ Période } & \multirow[b]{2}{*}{$\bar{Z}_{\max }$} & \multirow{2}{*}{$\begin{array}{l}t_{\mathrm{u}}-t_{\mathrm{sv}} \\
\text { en } \\
\text { minutes }\end{array}$} & \multicolumn{4}{|c|}{ Heures séparées (H.S.) } & \multicolumn{3}{|c|}{ Heures confondues } \\
\hline & & & $t=6^{\mathrm{h}}$ & $t=9^{\mathrm{h}}$ & $t=12^{\mathrm{h}}$ & $t=15^{\mathrm{h}}$ & $\rho$ & $\begin{array}{l}\sigma_{1}^{2} \\
\sigma_{3}^{2}\end{array}$ & $\begin{array}{l}\sigma_{2}^{2}, x_{t} \\
\sigma_{4}^{2}\end{array}$ \\
\hline $\begin{array}{r}5 \text { janvier } \\
26 \text { janvier }\end{array}$ & 53 & 1 & - & $\begin{array}{l}0,84 \\
0,20, h\end{array}$ & $\begin{array}{l}0,83 \\
0,24, p\end{array}$ & $\begin{array}{l}0,82 \\
0,29, T\end{array}$ & 0,84 & $\begin{array}{l}0,30 \\
0,25\end{array}$ & $\begin{array}{l}0,30, z_{t-1} \\
0,24\end{array}$ \\
\hline $\begin{array}{l}5 \text { février } \\
25 \text { février }\end{array}$ & 91 & 6 & - & $\begin{array}{l}0,89 \\
0,20, z\end{array}$ & $\begin{array}{l}0,83 \\
0,27, h\end{array}$ & $\begin{array}{l}0,82 \\
0,32, z\end{array}$ & 0,85 & $\begin{array}{l}0,28 \\
0,27\end{array}$ & $\begin{array}{l}0,28, h_{t} \\
0,26\end{array}$ \\
\hline $\begin{array}{l}1 \text { mars } \\
20 \text { mars }\end{array}$ & 130 & 2 & - & $\begin{array}{l}0,89 \\
0,18, T\end{array}$ & $\begin{array}{l}0,86 \\
0,23, h\end{array}$ & $\begin{array}{l}0,81 \\
0,29, h\end{array}$ & 0,86 & $\begin{array}{l}0,26 \\
0,24\end{array}$ & $\begin{array}{l}0,26, h_{t} \\
0,22\end{array}$ \\
\hline $\begin{array}{r}1 \text { avril } \\
21 \text { avril }\end{array}$ & 174 & -7 & - & $\begin{array}{l}0,86 \\
0,21, p\end{array}$ & $\begin{array}{l}0,82 \\
0,28, h\end{array}$ & $\begin{array}{l}0,81 \\
0,31, z\end{array}$ & 0,83 & $\begin{array}{l}0,31 \\
0,27\end{array}$ & $\begin{array}{l}0,29, h_{t} \\
0,27\end{array}$ \\
\hline $\begin{array}{l}1 \mathrm{mai} \\
19 \mathrm{mai}\end{array}$ & 197 & -11 & - & $\begin{array}{l}0,86 \\
0,22, T\end{array}$ & $\begin{array}{l}0,83 \\
0,28, z\end{array}$ & $\begin{array}{l}0,76 \\
0,35, p\end{array}$ & 0,81 & $\begin{array}{l}0,33 \\
0,28\end{array}$ & $\begin{array}{l}0,32, p_{t} \\
0,27\end{array}$ \\
\hline $\begin{array}{l}10 \text { juin } \\
30 \text { juin }\end{array}$ & 212 & -7 & $\begin{array}{l}0,86 \\
0,21, h\end{array}$ & $\begin{array}{l}0,84 \\
0,24, T\end{array}$ & $\begin{array}{l}0,81 \\
0,32, h\end{array}$ & $\begin{array}{l}0,78 \\
0,34, h\end{array}$ & 0,82 & $\begin{array}{l}0,32 \\
0,28\end{array}$ & $\begin{array}{l}0,32, h_{t} \\
0,27\end{array}$ \\
\hline $\begin{array}{l}22 \text { juillet } \\
11 \text { août }\end{array}$ & 211 & -2 & $\begin{array}{l}0,80 \\
0,22, p\end{array}$ & $\begin{array}{l}0,84 \\
0,26, T\end{array}$ & $\begin{array}{l}0,81 \\
0,30, z\end{array}$ & $\begin{array}{l}0,76 \\
0,38, p\end{array}$ & 0,80 & $\begin{array}{l}0,35 \\
0,29\end{array}$ & $\begin{array}{l}0,35, p_{t} \\
0,27\end{array}$ \\
\hline $\begin{array}{l}12 \text { août } \\
1 \text { septembre }\end{array}$ & 196 & -5 & - & $\begin{array}{l}0,87 \\
0,22, p\end{array}$ & $\begin{array}{l}0,78 \\
0,37, p\end{array}$ & $\begin{array}{l}0,78 \\
0,33, h\end{array}$ & 0,81 & $\begin{array}{l}0,34 \\
0,31\end{array}$ & $\begin{array}{l}0,34, p_{t} \\
0,30\end{array}$ \\
\hline $\begin{array}{l}10 \text { septembre } \\
30 \text { septembre }\end{array}$ & 158 & -15 & - & $\begin{array}{l}0,83 \\
0,26, h\end{array}$ & $\begin{array}{l}0,77 \\
0,35, h\end{array}$ & $\begin{array}{l}0,83 \\
0,28, h\end{array}$ & 0,81 & $\begin{array}{l}0,33 \\
0,30\end{array}$ & $\begin{array}{l}0,33, h_{t} \\
0,30\end{array}$ \\
\hline $\begin{array}{l}10 \text { octobre } \\
30 \text { octobre }\end{array}$ & 112 & -23 & - & $\begin{array}{l}0,89 \\
0,15, h\end{array}$ & $\begin{array}{l}0,85 \\
0,21, p\end{array}$ & $\begin{array}{l}0,84 \\
0,26, h\end{array}$ & 0,86 & $\begin{array}{l}0,26 \\
0,21\end{array}$ & $\begin{array}{l}0,21, h_{t} \\
0,21\end{array}$ \\
\hline $\begin{array}{l}11 \text { novembre } \\
30 \text { novembre }\end{array}$ & 61 & -22 & - & $\begin{array}{l}0,86 \\
0,17, p\end{array}$ & $\begin{array}{l}0,87 \\
0,18, h\end{array}$ & $\begin{array}{l}0,80 \\
0,31, h\end{array}$ & 0,84 & $\begin{array}{l}0,28 \\
0,22\end{array}$ & $\begin{array}{l}0,27, h_{t} \\
0,22\end{array}$ \\
\hline
\end{tabular}

where $\sigma_{1}^{2}, \sigma_{2}^{2}, \sigma_{3}^{2}, \sigma_{4}^{2}$ are defined through equations(1),(2),(3),(4).] 
$\tau_{t}, p_{t}, z_{t-1}$ dans une régression pas à pas ascendante. Une étude préliminaire a montré que l'introduction de ces quatre variables donne souvent une estimation sans biais de la variance résiduelle d'une valeur supérieure à celle obtenue avec une seule, $x_{r}$. Nous nous sommes donc limités à un modèle de type (2).

Le tableau II donne pour diverses périodes de l'année, et à heure fixée $t$, l'estimée de l'autocorrélation $\rho\left(z_{t}, z_{t+1}\right)$ ainsi que la variable $x_{t}$ sélectionnée et l'écart-type résiduel $\sigma_{2}$ des résidus de l'équation (2). Cette variable $x_{t}$ choisie est le plus souvent l'humidité $h_{t}$ mais de justesse devant la pression $p_{t}$, plus rarement $z_{t-1}$ qui est généralement non significatif. L'écarttype $\sigma_{1}$ des résidus de (1) est $\sigma_{1}=\sqrt{1-\rho^{2}}$. On remarque dans ce tableau la relative stabilité, au cours de l'année, d'un modèle prenant en compte les variables réduites, ce qui avait déjà été noté dans le cas des études à une seule variable [1, 2], ainsi la tendance générale à une légère décroissance de l'autocorrélation $\rho_{t}$ au cours de la journée, les différences entre valeurs successives restant toutefois dans les limites des intervalles de confiance. Avec une bonne approximation, on peut considérer que l'ensoleillement $\left(z_{t}\right)$ forme un processus autorégressif simple $\operatorname{AR}(1)$ : $z_{t+1}=\rho z_{t}+\varepsilon_{t}$ sans intervention des variables exogènes, mais dont le coefficient $\rho$ décroît très lentement du matin au soir.

3.2 RÉGRESSIONS A HEURES CONFONDUES (H.C.). Les régressions précédentes sont comparées à un modèle global (test F) où on ne différencie plus les valeurs de l'heure $t$ dans la journée. On a alors un échantillon de taille $252 \times 4=1004$. Le test $F$ donne des valeurs non significatives, et les autocorrélations des résidus $\varepsilon_{t}$ du modèle

$$
z_{t}=\rho z_{t-1}+\varepsilon_{t}
$$

peuvent être considérées comme nulles. Ceci confirme les résultats obtenus par Boch, Boileau, Bénard [2].

Dans ce même tableau II figurent : dans la colonne "H.C. ", la valeur estimée de $\rho$, et dans la dernière colonne les variances résiduelles estimées $\sigma_{1}^{2}, \sigma_{2}^{2}, \sigma_{3}^{2}$, $\sigma_{4}^{2}$ de chacun des modèles :

$$
\begin{aligned}
z_{t+1} & =\rho z_{t}+\varepsilon_{1, t+1} & & \sigma_{1}^{2}=1-\rho^{2}=\operatorname{var} \varepsilon_{1} \\
z_{t+1} & =a z_{t}+b x_{t}+\varepsilon_{2, t+1} & & \sigma_{2}^{2}=\operatorname{var} \varepsilon_{2} \\
z_{t+1} & =a z_{t}+b x_{t}+c n_{t}+\varepsilon_{3, t+1} & & \sigma_{3}^{2}=\operatorname{var} \varepsilon_{3} \\
z_{t+1} & =a z_{t}+c n_{t}+d n_{t+3}+\varepsilon_{4, t+1} & & \sigma_{4}^{2}=\operatorname{var} \varepsilon_{4}
\end{aligned}
$$

où $n_{t}$ est la nébulosité (centrée). Nous avons introduit le couple $\left(n_{t}, n_{t+3}\right)$ qui caractérise, d'une certaine façon, la stabilité du temps sur trois heures.

Pour les mois de mai à septembre, on peut avec une bonne approximation considérer que $\rho=0,81$. Les mois de saison plus froide donnent une autocorrélation $\rho$ un peu plus élevée $(0,84)$ (voir également [2], Fig. 4).

La seule variable qui, en plus de $z_{t}$, améliore la prévision de $z_{t+1}$ est la nébulosité $n_{t}$. Dans le meilleur des cas, elle fait passer l'écart-type résiduel de $\sigma_{1}=0,55$ à $\sigma_{3}=0,50$, soit un gain de $10 \%$. Mais $n_{t}$ est mesurée sans appareil de mesure et la seule prévision «automatique " de $z_{t+1}$ à l'heure $t$ est :

- de mai à septembre : $\hat{z}_{t+1}=0,81 z_{t}$;

- d'octobre à avril : $\hat{z}_{t+1}=0,84 z_{t}$.

Les essais d'introduction des variables explicatives dans la régression, prises à l'instant $t-1$, pour faire intervenir les variations de ces variables entre les instants $t-1$ et $t$ n'ont toujours abouti qu'à des résultats non significatifs.

4. Conditionnement par le signe de $z_{\boldsymbol{t}^{*}}-$ Pour tenir compte de la stabilité du temps (voir [2]) on considère la corrélation entre les variables réduites définies précédemment $z_{t}$ et $z_{t+1}$ conditionnée par le signe de $z_{t}$. On estime :

$$
\begin{aligned}
& \rho^{+}(t)=\operatorname{corr}\left(z_{t}, z_{t+1} / z_{t}>0\right) \\
& \rho^{-}(t)=\operatorname{corr}\left(z_{t}, z_{t+1} / z_{t} \leqslant 0\right) .
\end{aligned}
$$

On détermine également les équations de régression

$$
z_{t+1}=a+b z_{t}+\varepsilon_{t+1}
$$

où $a$ et $b$ dépendent du signe de $z_{t}$. On obtient ainsi pour chaque cas, les écarts-types résiduels estimés $\sigma_{\varepsilon, t}^{+}$et $\sigma_{\varepsilon, t^{-}}^{-}$Les résultats, pour la période du $10 \mathrm{au}$ 30 juin (Fig. 1) montrent une corrélation toujours plus élevée lorsque $z_{t} \leqslant 0$. Mais comme les écartstypes de $z_{t}$ et de $z_{t+1}$ conditionnés par le signe de $z_{t}$ sont différents, ils se trouvent cependant que $\sigma_{\varepsilon, t}^{-}>\sigma_{\varepsilon, t^{*}}^{+}$.

Pour la période étudiée ( 10 au 30 juin), la fréquence de passage à l'intérieur d'une même journée de $z_{t}>0$ à $z_{t+1} \leqslant 0$ est de 0,14 et de $z_{t} \leqslant 0$ à $z_{t+1}>0$, de 0,18 . Le beau temps semble donc être légèrement plus stable.

Les autocorrélations $\rho^{+}$et $\rho^{-}$sont ensuite calculées sans différencier les heures entre elles. On trouve :

$$
\rho^{+}=0,51, \quad \rho^{-}=0,65 .
$$



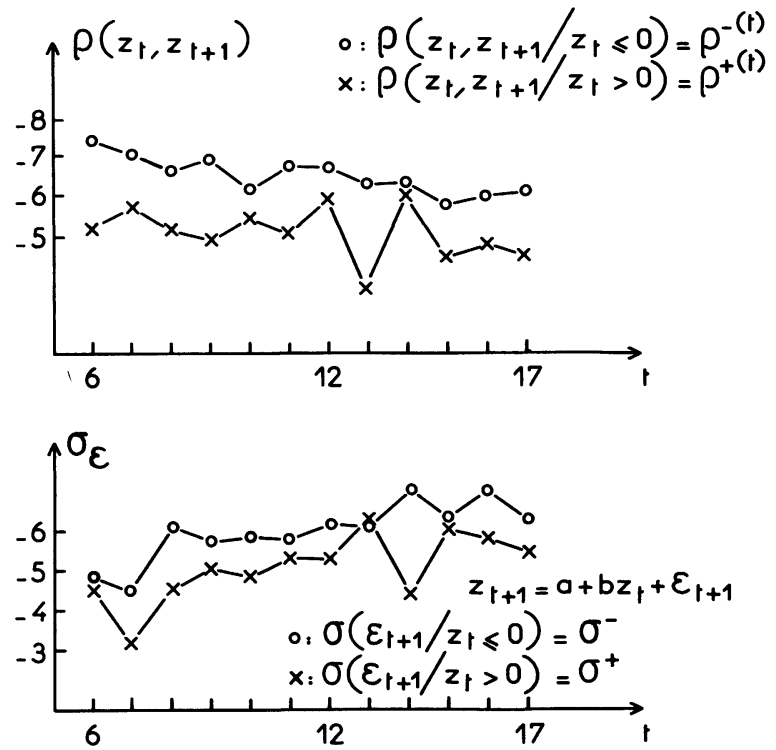

Fig. 1. - Autocorrélation et écart-type résiduels conditionnellement au signe de $z_{t}$ (du 10 au 30 juin).

[Residual autocorrelation and standard deviation conditional to the sign of $z_{t}$ (from the 10th to the 30th of June).]

Les équations de régression sont :

pour $z_{t}>0$ :

$$
z_{t+1}=0,03+0,78 z_{t}+\varepsilon_{t+1} ; \quad \sigma_{\varepsilon}^{+}=0,52
$$

pour $z_{t} \leqslant 0$ :

$$
z_{t+1}=0,07+0,89 z_{t}+\varepsilon_{t+1} ; \quad \sigma_{\varepsilon}^{-}=0,60 .
$$

Rappelons que, sans ce conditionnement, on avait $\sigma_{\varepsilon}=0,57$. Le conditionnement n'apporte donc pas grand-chose.

Le tableau III donne les résultats de prévisions au pas de temps horaire à partir de données du mois de juin 1965. Les valeurs S1 sont les données vraies de l'irradiation, les valeurs $\mathrm{S} 2$ les données prévues par :

$$
\hat{z}_{t+1}=0,82 z_{t} \text { puis } \hat{Z}_{t+1}=\sigma\left(Z_{t+1}\right) \cdot \hat{z}_{t+1}+\bar{Z}_{t+1},
$$

les valeurs $\mathrm{S} 3$ sont les données prévues par les équations ci-dessus en différenciant $z_{t}>0$ et $z_{t} \leqslant 0$. On voit que S3 n'apporte ni mieux ni pire que $S 2$. La prise en compte de la stabilité du temps est difficile. On a certes affaire en première approximation à un processus AR(1), mais (cf. [2]) les autocorrélations $\rho_{i}=\rho\left(z_{t}, z_{t-i}\right)$ ne tendent pas vers zéro comme elles le devraient dans le cas d'un véritable processus AR(1) pour $z_{t}$. Le conditionnement par le signe de $\sum_{i} z_{t-i}$ sur quelques heures précédentes [2] ne donnent pas de meilleurs résultats que ceux de ce paragraphe. Il y a sans doute des progrès à réaliser pour faire intervenir la mémoire sur un temps plus long. Peutêtre en conditionnant l'autocorrélation non par la somme des ensoleillements précédents mais par la

Tableau III. - Prévision horaire pour des données de juin 1965. S1 : données réelles; $\mathrm{S} 2: \hat{z}_{t+1}=0,82 z_{t} ; \mathrm{S} 3$ : séparation $\left(z_{t}>0\right)\left(z_{t} \leqslant 0\right)$.

[Hourly forecast the data of June 1965. S1 : real data; S2 : $\hat{z}_{t+1}=0.82 z_{t} ; \mathrm{S} 3:$ separation between $z_{t}>0$ and $z_{t} \leqslant 0$.]

Heure $t$

\begin{tabular}{|c|rrrrrrrrrrrrr|}
\hline $\bar{Z}_{t}$ & 35 & 71 & 112 & 152 & 180 & 198 & 208 & 210 & 202 & 184 & 149 & 115 & 74 \\
\hline$\sigma\left(Z_{t}\right)$ & 18 & 35 & 53 & 65 & 75 & 83 & 86 & 84 & 83 & 74 & 64 & 48 & 32 \\
\hline S1 & 14 & 32 & 60 & 137 & 197 & 268 & 260 & 250 & 89 & 208 & 196 & 197 & 85 \\
\hline S2 & & 38 & 64 & 100 & 165 & 213 & 267 & 252 & 235 & 101 & 166 & 143 & 119 \\
\hline S3 & & 37 & 64 & 100 & 169 & 215 & 266 & 253 & 276 & 99 & 168 & 143 & 118 \\
\hline S1 & 14 & 16 & 65 & 70 & 104 & 135 & 195 & 255 & 276 & 245 & 197 & 130 & 89 \\
\hline S2 & & 38 & 45 & 105 & 102 & 129 & 154 & 200 & 239 & 237 & 193 & 144 & 83 \\
\hline S3 & & 37 & 43 & 105 & 100 & 129 & 156 & 205 & 239 & 237 & 193 & 144 & 83 \\
\hline S1 & 41 & 50 & 85 & 171 & 192 & 250 & 339 & 360 & 329 & 287 & 176 & 99 & 80 \\
\hline S2 & 81 & 86 & 125 & 198 & 208 & 252 & 316 & 323 & 276 & 223 & 131 & 66 \\
\hline S3 & 82 & 87 & 127 & 199 & 211 & 252 & 313 & 320 & 274 & 221 & 131 & 67 \\
\hline
\end{tabular}


valeur du temps de séjour dans l'état $\left(z_{t}>0\right)$ ou $\left(z_{t} \leqslant 0\right)$. C'est un peu en ce sens que nous avons orienté les études de simulation ci-après $(\S 6)$, en considérant les temps de séjour dans un état de nébulosité donné.

5. Conditionnement par la nébulosité. - 5.1. La nébulosité est mesurée toutes les trois heures par un entier entre 0 (nébulosité nulle) et 8 . Nous considérons la variable d'irradiation intégrée entre $t-1$ et $t, Z_{t}$ et construisons comme ci-dessus la variable réduite $z_{t}=\left(Z_{t}-\bar{Z}_{t}\right) / \sigma\left(Z_{t}\right)$. Pour les heures $t=6$, 9,12 ou 15 et une valeur $x$ fixée pour la nébulosité $n_{t}$, on considère tous les vecteurs $\left(n_{t}, z_{t}, z_{t+1}, z_{t+2}\right)$ et on suppose que les relations entre $z_{t}, z_{t+1}$ et $z_{t+2}$ sont indépendantes de $t$. Sont alors estimées :

- les moyennes conditionnelles $\mathrm{E}\left(z_{t+1} / n_{t}=x\right)$;

- les écarts-types, médianes et autocorrélations conditionnels.

Les médianes diffèrent. généralement très peu des moyennes. Les résultats figurent sur la figure 2. Les courbes des moyennes restent pratiquement identiques pour les périodes étudiées d'avril à septembre, ainsi que celles des écarts-types qui, globalement, croissent avec la nébulosité. En revanche le comportement des autocorrélations conditionnelles est moins régulier.

5.2 TYPE DE TEMPS. - A partir de ces résultats, les neuf valeurs des nébulosités $n_{t}$ sont regroupées en trois valeurs $\tilde{n}_{t}$ :

$$
\begin{gathered}
\text { nébulosité faible }: \tilde{n}_{t}=1: n_{t}=0,1,2,3 \\
\text { - moyenne }: \tilde{n}_{t}=2: n_{t}=4,5,6 \\
\text { forte } \quad: \quad \tilde{n}_{t}=3: n_{t}=7,8 .
\end{gathered}
$$

On étudie alors l'évolution des autocorrélations suivant l'évolution du type de nébulosité, c'est-à-dire qu'on estime des grandeurs statistiques conditionnées par le couple $\left(\tilde{n}_{t}, \tilde{n}_{t+3}\right)$.

5.2.1 Temps stable. - Les couples respectifs $\left(\tilde{n}_{t}, \tilde{n}_{t+3}\right)=(1,1),(2,2)$ ou $(3,3)$ définissent des « beaux temps", "temps couvert», "mauvais temps" (Tableau IV).

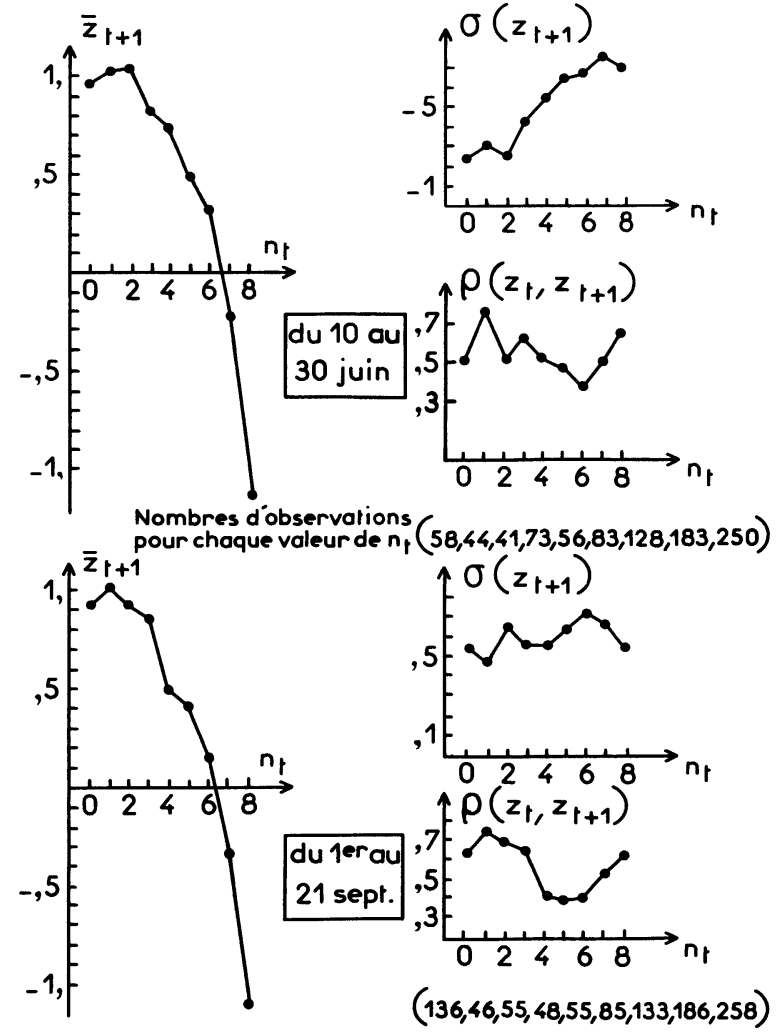

Fig. 2. - Irradiation réduite moyenne, écart-type et autocorrélation en fonction de la nébulosité $n_{t}$.

[Mean reduced irradiation, standard deviation and autocorrelation as functions of the nebulosity $n_{t}$.]

Pour chacun de ces termes caractérisant un « temps stable " on observe une relative stabilité des quantités calculées (moyennes, écarts-types, autocorrélations) qui montre :

a) une croissance de l'écart-type du bon ou mauvais temps (Tableau IV), évidente si on se reporte aux courbes initiales en fonction de $n_{t}$ (Fig. 2),

b) une autocorrélation faible pour $\left(\tilde{n}_{t}, \tilde{n}_{t+3}\right)=(2,2)$, de l'ordre de 0,45 et plus forte pour les " beaux " et " mauvais » temps (Tableau IV).

Tableau IV. - Moyenne, écart-type et autocorrélation de l'irradiation réduite par « temps stable».

[Mean-value, standard deviation and autocorrelation of the reduced irradiation with « steady weather ».]

\begin{tabular}{|l|c|c|c|}
\hline \multicolumn{4}{|c|}{ Période du 10 au 30 juin } \\
\hline \multicolumn{1}{|c|}{$\left(\tilde{n}_{t}, \tilde{n}_{t+3}\right)$} & $(1,1)$ & $(2,2)$ & $(3,3)$ \\
\hline $\begin{array}{l}\text { Nombre } \\
\text { de données }\end{array}$ & 135 & 146 & 327 \\
\hline $\bar{z}_{t+1}$ & 0,98 & 0,48 & $-0,93$ \\
\hline$\sigma\left(z_{t+1}\right)$ & 0,30 & 0,65 & 0,80 \\
\hline$\rho\left(z_{t+1}, z_{t+2}\right)$ & 0,72 & 0,50 & 0,75 \\
\hline
\end{tabular}

\begin{tabular}{|l|c|c|c|}
\hline \multicolumn{4}{|c|}{ Période du 1er au 21 septembre } \\
\hline \multicolumn{1}{|c|}{$\left(\tilde{n}_{t}, \tilde{n}_{t+3}\right)$} & $(1,1)$ & $(2,2)$ & $(3,3)$ \\
\hline $\begin{array}{l}\text { Nombre de } \\
\text { de données }\end{array}$ & 210 & 141 & 321 \\
\hline $\bar{z}_{t+1}$ & 0,93 & 0,40 & $-0,93$ \\
\hline$\sigma\left(z_{t+1}\right)$ & 0,54 & 0,65 & 0,68 \\
\hline$\rho\left(z_{t+1}, z_{t+2}\right)$ & 0,75 & 0,38 & 0,70 \\
\hline
\end{tabular}


5.2.2 Transition. - Lors des passages d'un type de nébulosité à un autre, les autocorrélations $\rho$ de $z$ sont mal définies et ne semblent pas indiquer de résultat d'ensemble, si ce n'est : $0,30 \leqslant \rho \leqslant 0,65$.

Ces autocorrélations sont généralement calculées sur une soixantaine d'observations; donc avec un écart-type de l'ordre de 0,15 .

L'évolution des moyennes $\bar{z}_{t+1}, \bar{z}_{t+2}, \bar{z}_{t+3}$, conditionnées par $\left(\tilde{n}_{t}, \tilde{n}_{t+3}\right)$ est donnée par les tableaux V. Mais là encore les écarts-types des observations ne sont pas constants.

Les courbes de la figure 2 et les tableaux IV et V sont reportés pour deux périodes : 10 au 30 juin et 1 er au 21 septembre. Pour d'autres périodes, on obtient des résultats tout à fait semblables.

Tableau V. - Evolution de la moyenne de l'irradiation réduite par temps instable, caractérisé par $\left(\tilde{n}_{t} \rightarrow \tilde{n}_{t+3}\right)$. $\sigma$ moyen est l'écart-type de $z_{t+2}$ et $N_{\mathrm{obs}}$ le nombre d'observations considéré.

[Evolution of the mean-value of the reduced irradiation for unsteady weather, characterized by $\left(\tilde{n}_{t} \rightarrow \tilde{n}_{t+3}\right)$. " $\sigma$ moyen " is the standard deviation of $z_{t+2}$ and $N_{\text {obs }}$ the number of observations that are taken into account.]

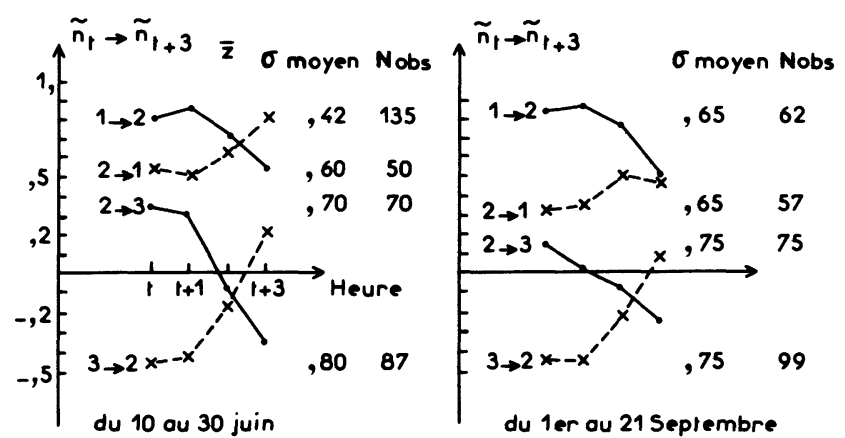

6. Simulation au pas de temps horaire. - 6.1 EvoLUTION DE LA NÉBULOSITÉ. - Comme nous venons de le voir, la nébulosité conditionne fortement l'ensoleillement. Ce résultat est utilisé pour construire un modèle de simulation de l'ensoleillement à partir d'une simulation de la nébulosité de 6 à 18 heures au pas de temps trihoraire, sur trois semaines, Les nébulosités $n_{t}$ sont regroupées comme précédemment suivant trois états $\tilde{n}_{t}=1,2,3$. La simulation est faite à partir des résultats statistiques du 10 au 30 juin. Afin de construire le modèle de simulation de la nébulosité, on détermine les matrices de transition aux heures $t=6,9,12,15$. Les matrices $3 \times 3$, $P(t)=\left(p_{i j}(t)\right)$ ont pour éléments les probabilités de passage de l'état de nébulosité $i$ à l'instant $t$ à l'état de nébulosité $j$ à l'instant $t+3$ :

$$
p_{i j}(t)=P\left(\tilde{n}_{t+3}=j / \tilde{n}_{t}=i\right) .
$$

On a ainsi la matrice $P(t)=\left(p_{i j}(t)\right)$, déterminée à l'aide des fréquences observées.

On définit la matrice de transition $P$ où les fréquences sont estimées indépendamment de l'heure :

$$
P=\left[\begin{array}{lll}
0,62 & 0,31 & 0,07 \\
0,20 & 0,55 & 0,26 \\
0,06 & 0,20 & 0,74
\end{array}\right] .
$$

Le CHI-deux testant l'hypothèse d'égalité des quatre matrices [8] a une valeur de 40 pour 18 degrés de liberté. On a donc un CHI-deux significatif, mais néanmoins pas "catastrophique". Les estimations sur d'autres périodes de l'année donnent des résultats analogues : en principe les fréquences de transition des nébulosités évoluent au cours de la journée.

D'autre part, l'hypothèse d'une chaîne de Markov homogène pour l'évolution de $\tilde{n}_{t}$ sous-estime la stabilité des états. En effet, sous l'hypothèse markovienne, la distribution du temps de séjour $T_{i}$ dans un état $i$ donné devrait être géométrique : du type $P\left(T_{i}=k\right)=C a^{k}$. Or le tableau VII montre clairement que les distributions des temps de séjour dans les états sont non-géométriques.

Enfin la matrice $Q$ de transition entre $18^{\mathrm{h}}$ et $6^{\mathrm{h}}$ le lendemain est estimée et comparée à la puissance quatrième de $P$.

$$
\begin{aligned}
Q_{18-6} & =\left[\begin{array}{lll}
0,55 & 0,22 & 0,23 \\
0,32 & 0,30 & 0,38 \\
0,10 & 0,25 & 0,65
\end{array}\right] ; \\
P^{4} & =\left[\begin{array}{lll}
0,35 & 0,35 & 0,30 \\
0,27 & 0,34 & 0,39 \\
0,20 & 0,30 & 0,50
\end{array}\right] .
\end{aligned}
$$

On voit qu'une hypothèse markovienne sous-estimerait la stabilité des états 1 et 3 : sous l'hypothèse

Tableau VI. - Matrices de transition pour les trois états de nébulosité.

[Transition matrices for the three states of nebulosity.]

\begin{tabular}{c|cccc}
$t$ & \multicolumn{3}{|c}{6 heures } & \multicolumn{3}{c}{9 heures } & \multicolumn{1}{c}{12 heures } \\
\hline \multirow{3}{*}{$P(t)$} & {$\left[\begin{array}{llll}0,64 & 0,30 & 0,06 \\
0,23 & 0,44 & 0,33 \\
0,05 & 0,18 & 0,77\end{array}\right] \quad\left[\begin{array}{lll}0,48 & 0,42 & 0,10 \\
0,10 & 0,54 & 0,36 \\
0,02 & 0,18 & 0,80\end{array}\right] \quad\left[\begin{array}{llll}0,74 & 0,26 & 0,0 \\
0,10 & 0,64 & 0,26 \\
0,07 & 0,20 & 0,73\end{array}\right] \quad\left[\begin{array}{llll}0,70 & 0,25 & 0,05 \\
0,31 & 0,52 & 0,17 \\
0,10 & 0,25 & 0,65\end{array}\right]$}
\end{tabular}


Tableau VII. - Distribution des temps de séjour dans les états.

[Distribution of the lifetimes of the states.]

\begin{tabular}{|c|cccccc|}
\hline \multirow{2}{*}{ Etat $\tilde{n}_{6}$} & \multicolumn{5}{|c|}{ Fréquence des temps de séjour $T$ dans l'état $\tilde{n}_{6}$} \\
(à 6 heures) & $T=0$ & $T=3$ & $T=6$ & $T=9$ & $T \geqslant 12$ & Nombre \\
\hline 1 & 0,36 & 0,31 & 0,08 & 0,08 & 0,17 & 72 \\
2 & 0,56 & 0,24 & 0,13 & 0,07 & 0,00 & 45 \\
3 & 0,23 & 0,15 & 0,14 & 0,14 & 0,34 & 112 \\
\hline
\end{tabular}

markovienne, la matrice de transition $Q$ en 4 étapes ( 4 triheures) devrait être égale à la puissance quatrième de celle en une étape, $Q=P^{4}$.

6.2 Simulation DE L'ÉVOLUTION DE LA NÉBUlOSITÉ (10 AU 30 JUIN). - Les résultats précédents sont utilisés pour simuler les valeurs des nébulosités $\tilde{n}_{t}(=1,2$ ou 3$)$ sur 21 jours aux heures $t=6,9,12,15$, 18. Une première valeur est tirée au sort à 6 heures le premier jour. Les fréquences cumulées des temps de séjour dans un état $i$ sont entrées comme données, ainsi que les probabilités de transition (indépendantes de $t)$ de $\left(\tilde{n}_{t}=i\right)$ à $\left(\tilde{n}_{t+3}=j\right)$ lorsqu'il y a changement d'état. Ceci est déduit de la matrice $P$.

On fait donc l'hypothèse que les temps de séjour ont même distribution à partir de chaque changement d'état et que les probabilités de transition ne changent pas dans la journée. Il ne s'agit évidemment là que d'un pis-aller simplificateur, vu la croissance très rapide du nombre de paramètres à estimer dès que l'on veut affiner le modèle. On aboutit ainsi à un modẹle semi-markovien où les temps de séjour dans un état sont des variables aléatoires de lois connues et où, indépendamment, les changements d'état se font suivant des fréquences de transition connues. Ces lois de probabilités sont construites à partir des fréquences estimées sur les données disponibles.

Pour passer de la valeur $\tilde{n}_{18}$ en fin de journée à la valeur $\tilde{n}_{6}$ du lendemain, on utilise la matrice de transition $Q_{18-6}$ estimée ci-dessus. On obtient ainsi pour chaque journée durant trois semaines, cinq valeurs simulées des nébulosités toutes les trois heures, de 6 à 18 heures. Un couple $\left(\tilde{n}_{t}, \tilde{n}_{t+3}\right)$ définit alors, pour la simulation de l'irradiation globale entre $t$ et $t+3$ le type de temps dans lequel on est placé.

6.3 Simulation de L'irradiation. - Pour chaque couple fixé $\left(\tilde{n}_{t}, \tilde{n}_{t+3}\right)=(i, j)$, on suppose connus pour les irradiations réduites $z_{t}, z_{t+1}, z_{t+2}, z_{t+3}$ les quatre moyennes $m_{i j}(t) \ldots m_{i j}(t+3)$ ainsi que l'autocorrélation $\rho_{i j}$ et l'écart-type $\sigma_{i j}$ supposés constants entre les instants $t$ et $t+3$.

Avec ces données, l'irradiation réduite $z_{t}$ est simulée suivant l'équation : $\left(z_{t}-m_{t}\right)=\rho_{t}\left(z_{t-1}-m_{t-1}\right)+\sigma_{t} \varepsilon_{t}$ où $m_{t-1}, m_{t}, \rho_{t}$ et $\sigma_{t}$ sont lues dans le tableau IX en fonction des nébulosités trihoraires précédentes et suivantes. Le processus $\left(\varepsilon_{t}\right)$ est un bruit blanc $N(0,1)$,
Tableau VIII. - Données pour la simulation des nébulosités de 6 à 18 heures, du 10 au 20 juin.

[Data for the simulation of the nebulosities from 6 AM to 6 PM (10-20 of June).]

1. Fréquences des temps de séjour $T$ dans un état (triheures)

\begin{tabular}{|c|c|c|c|c|c|}
\hline$T$ & 0 & 1 & 2 & 3 & $\geqslant 4$ \\
\hline$\tilde{n}_{t}=1$ & 0,35 & 0,30 & 0,10 & 0,10 & 0,15 \\
$\tilde{n}_{t}=2$ & 0,55 & 0,25 & 0,15 & 0,05 & 0,00 \\
$\tilde{n}_{t}=3$ & 0,25 & 0,15 & 0,15 & 0,15 & 0,30 \\
\hline
\end{tabular}

2. Probabilités de transition d'un état à un autre

\begin{tabular}{|c|c|c|c|}
\hline$\tilde{n}_{t}$ & 1 & 2 & 3 \\
\hline 1 & - & 0,8 & 0,2 \\
\hline 2 & 0,5 & - & 0,5 \\
\hline 3 & 0,2 & 0,8 & - \\
\hline
\end{tabular}

3. Matrice de transition de $18^{\mathrm{h}} \stackrel{a}{ } 6^{\mathrm{h}}$ : la matrice $Q_{18-6}$ ci-dessus.

mais les valeurs supérieures à 2 ou inférieures à -2 sont tronquées respectivement à 2 et -2 . La première donnée du jour, à 6 heures, est tirée au sort suivant $z_{6}=m_{6}+\sigma_{6} \varepsilon_{6}$, où $m_{6}$ et $\sigma_{6}$ correspondent aux valeurs du tableau IX pour $\left(\tilde{n}_{6}, \tilde{n}_{9}\right)$. On obtient ainsi, pour chaque jour, treize valeurs $z_{6}, z_{7}, \ldots, z_{18}$, à partir desquelles on construit l'irradiation globale $Z_{t}$ à l'heure $t$ :

$$
Z_{t}=\bar{Z}_{t}+S_{t} z_{t}
$$

$\bar{Z}_{t}$ et $S_{t}$ sont la moyenne et l'écart-type estimés sur l'irradiation globale à l'heure $t$. Elles sont données par le tableau $X$, pour la période étudiée. 
Tableau IX. - Moyennes, écart-type, autocorrélation des irradiations réduites pour un passage de $\left(\tilde{n}_{t}=i\right) \grave{a}$ $\left(\tilde{n}_{t+3}=j\right)$.

[Mean-values, standard deviations and autocorrelations of the reduced irradiation when passing from $\tilde{n}_{t}=i$ to $\tilde{n}_{t+3}=j$.]

\begin{tabular}{|l|l|l|l|}
\hline \multicolumn{1}{|c|}{1} & \multicolumn{1}{|c|}{2} & \multicolumn{1}{|c|}{3} \\
\hline 1 & $\begin{array}{l}m \equiv 1 \\
\rho=0,7 ; \quad \sigma=0,3\end{array}$ & $\begin{array}{l}m=0,9 ; 0,8 ; 0,7 ; 0,5 \\
\rho=0,5 ; \quad \sigma=0,4\end{array}$ & $\begin{array}{l}m=0,8 ; 0,3 ;-1,0 ;-0,4 \\
\rho=0,5 ; \quad \sigma=0,6\end{array}$ \\
\hline 2 & $\begin{array}{l}m=0,5 ; 0,5 ; 0,7 ; 0,9 \\
\rho=0,5 ; \quad \sigma=0,6\end{array}$ & $\begin{array}{l}m=0,5 \\
\rho=0,5 ; \quad \sigma=0,65\end{array}$ & $\begin{array}{l}m=0,4 ; 0,3 ;-0,1 ;-0,5 \\
\rho=0,5 ; \quad \sigma=0,7\end{array}$ \\
\hline 3 & $\begin{array}{l}m=-0,5 ;-0,1 ; 0,4 ; 0,8 \\
\rho=0,5 ; \quad \sigma=0,7\end{array}$ & $\begin{array}{l}m=-0,5 ;-0,4 ;-0,2 ; 0,2 \\
\rho=0,5 ; \quad \sigma=0,75\end{array}$ & $\begin{array}{l}m=0,9 \\
\rho=0,75 ; \quad \sigma=0,8\end{array}$ \\
\hline
\end{tabular}

Tableau X. - Moyenne $\bar{Z}_{t}$ et écart $S_{t}$ de l'irradiation globale $Z_{t} a \grave{a}$ l'heure $t$.

[Mean-value $\bar{Z}_{t}$ and deviation $S_{t}$ of the total solar irradiation $Z_{t}$ at time $t$.]

\begin{tabular}{|l|ccrrrrrrrrrrr|}
\hline$t$ & 6 & 7 & 8 & 9 & 10 & 11 & 12 & 13 & 14 & 15 & 16 & 17 & 18 \\
\hline $\bar{Z}_{t}$ & 35,0 & 71,0 & 112,0 & 152,0 & 180,0 & 198,0 & 208,0 & 210,0 & 202,0 & 184,0 & 149,0 & 115,0 & 74,0 \\
\hline$S_{t}$ & 18,0 & 35,0 & 53,0 & 65,0 & 75,0 & 83,0 & 85,0 & 84,0 & 83,0 & 74,0 & 64,0 & 47,0 & 32,0 \\
\hline
\end{tabular}

Les figures 3 et 4 représentent des données :

- entièrement simulées sur huit jours consécutifs : simulation des nébulosités puis des irradiations correspondantes (Fig. 3),

- simulées à partir de nébulosités correspondant
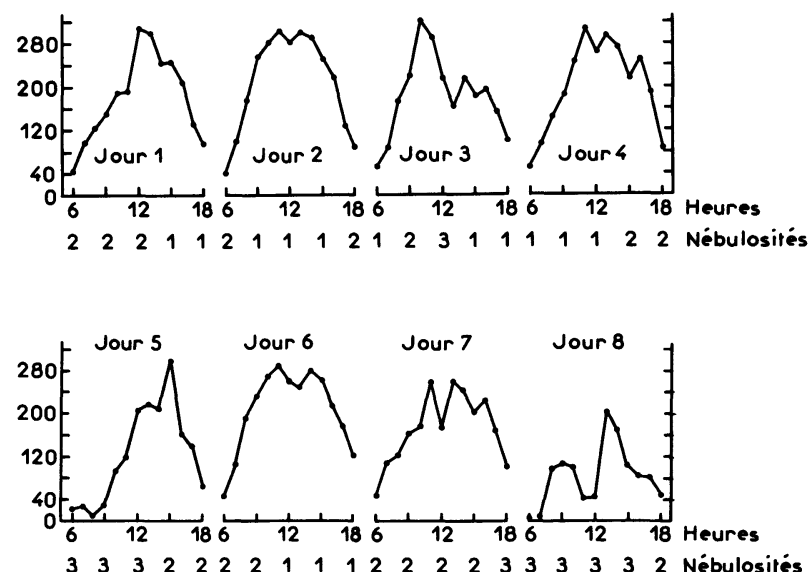

Fig. 3. - Données entièrement simulées sur huit jours consécutifs : simulation des nébulosités puis des irradiations correspondantes.

[Totally simulated data for eight following days : simulation of the nebulosities and of the corresponding irradiations.] à huit jours consécutifs en juin 1973. Ces simulations figurent en même temps que les données réelles (Fig. 4).

Les simulations montrent qu'on retrouve bien le comportement global d'évolution de l'irradiation. Toutefois dans le cas de très beau temps correspondant à des nébulosités mesurées nulles toute la journée, le fait de regrouper les nébulosités mesurées en trois groupes ne permet généralement pas de retrouver par simulation la régularité des courbes. On obtiendrait de meilleurs résultats s'il était possible de considérer les neuf états de la nébulosité. Pour les évolutions de " temps stable » (ex. $\left.n_{t}=n_{t+3}=0\right)$, les autocorrélations $\rho$ seraient meilleures et les écarts-types $\sigma$ plus faibles. Mais le nombre de fréquences à estimer augmente très vite : le passage de trois à neuf états de nébulosités fait passer le nombre de paramètres à estimer de 21 à 171 avec, pour les petites fréquences un nombre trop faible de données qui interdit toute estimation sérieuse. Le regroupement suivant les trois états ici définis nous a semblé le plus judicieux pour les données dont nous disposions. Des données d'un autre site pourraient imposer un regroupement différent.

7. Conclusion. - Pour un modèle de simulation, la prise en compte de la nébulosité comme variable conditionnant fortement l'irradiation permet d'intro- 


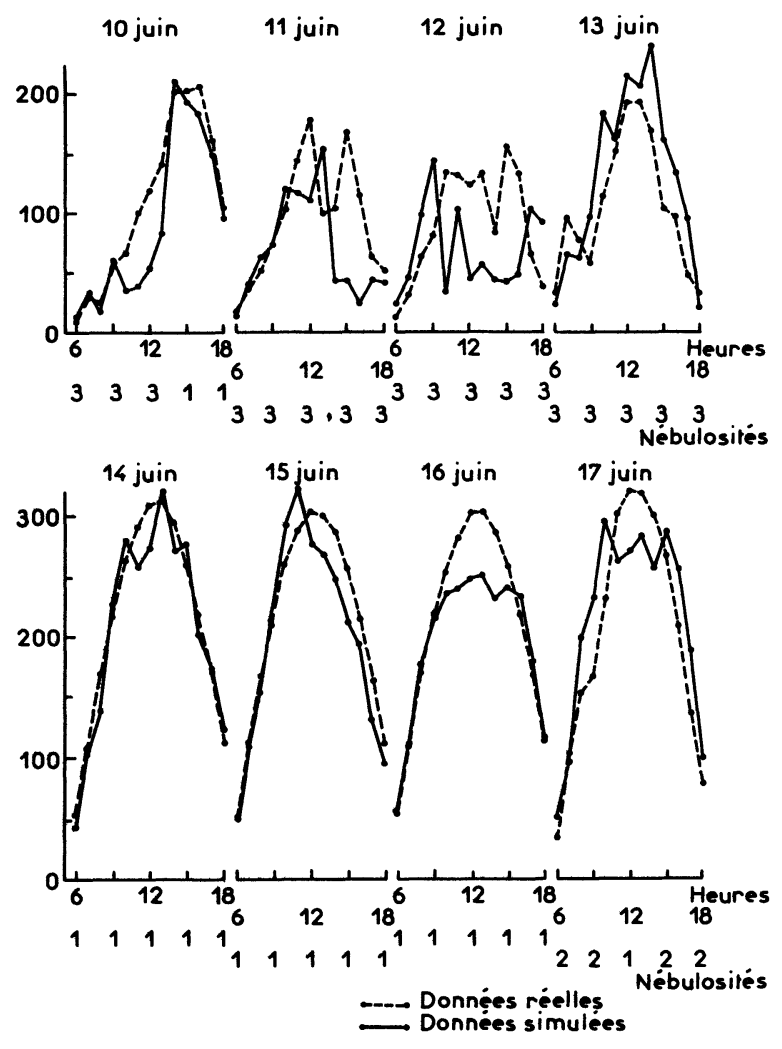

duire une plus grande stabilité du temps. Sur des sites où le temps est souvent beau mais sujet à de brusques variations, l'intérêt d'un tel modèle prenant en compte les temps de séjour et les probabilités de transition est évident.

Si on n'utilise que des variables mesurées localement à l'aide d'appareils de mesure (donc pas la nébulosité), un modèle de prévision se réduit pratiquement sur les irradiations réduites à un auto-régressif d'ordre 1 . Le modèle reste stable au cours de l'année, mais ne parvient pas à rendre compte de la stabilité du temps au cours de la journée, même en utilisant d'autres variables explicatives dans une équation de régression.

Fig. 4. - Données simulées à partir de nébulosités correspondant à huit jours consécutifs en jụin 1973. Ces simulations figurent en même temps que les données réelles.

[Data simulated from the nebulosities of eight consecutive days of June 1973. These simulations are plotted as well as the real data.]

\section{Bibliographie}

[1] Guerrier, B. Bolleau, E., Bénard, C., Analyse statistique temporelle de l'irradiation solaire globale quotidienne. Revue Phys. Appl. 15 (1980) 93-102.

[2] Boch, G., Bolleau, E., BéNARd, C., Modélisation de l'irradiation solaire au pas de temps de l'heure. Revue Phys. Appl. 16 (1981) 237-247.

[3] Box, G. E. P. and Jenkins, G. M., Time series analysis, forecasting and control (Holden Day) 1976.

[4] Bolleau, E., rapport interne LSS, Supélec, Plateau du Moulon, $91190 \mathrm{Gif} / \mathrm{s} /$ Yvette.
[5] Duhamel, Ch., Estimation d'une courbe de régression de la moyenne (programme REGMOY), Statistique et Analyse des Données 1-1978, p. 47-52.

[6] Cottrell, M., Duhamel, Ch., Genon Cathalot, V., Exercices de probabilités, Chap. VII (Belin) 1980.

[7] HAJEK, $A$ course in non-parametric statistics (Holden Day) 1969.

[8] Basawa, I. V., Rao, B. L. S., Statistical inference for stochastic processes (Academic Press) 1980. 